Supporting Information

\title{
Laccase-mediated Flavonoid Polymerization Leads to the Pericarp Browning of
}

\section{Litchi Fruit}

Junbin Wei ${ }^{1,2, \dagger}$, Xin Zhang ${ }^{1,2, \dagger}$, Ruihao Zhong ${ }^{1,2}$, Bin Liu ${ }^{1,2}$, Xuelian Zhang ${ }^{1,2}$, Fang Fang ${ }^{1,3}$, Zhaoqi Zhang ${ }^{1,3, *}$, Xuequn Pang1, 2, *

$\dagger$ These authors contributed equally to the article.

*Emails: Zhaoqi Zhang (zqzhang@scau.edu.cn); Xuequn Pang (xqpang@scau.edu.cn)

${ }^{1}$ State Key Laboratory for Conservation and Utilization of Subtropical Agro-bioresources / Guangdong Provincial Key Laboratory of Postharvest Science of Fruit and Vegetables /Engineering Research Center for Postharvest Technology of Horticultural Crops in South China, South China Agricultural University, Guangzhou, 510642, China

${ }^{2}$ College of Life Sciences, South China Agricultural University, Guangzhou, 510642, China

${ }^{3}$ College of Horticulture, South China Agricultural University, Guangzhou, 510642, China 
Table S1. List of primer used in this study

\begin{tabular}{ccc}
\hline Gene & Primer name & Sequence (5' to 3') \\
\hline \multirow{2}{*}{$A D E / L A C$} & Primers for Overexpression \\
& ADE/LAC-F & ATGGGTTTGGTGTCGAGGTTC \\
& ADE/LAC-R & TCAAGAGCTGGAACAACTAGGC \\
\hline \multirow{3}{*}{ AtActin7 } & Primers for Quantitative real-time PCR analysis \\
& qActin7-F & CCGGTATTGTGCTCGATTCTG \\
ADE/LAC & qActin7-R & TTCCCGTTCTGCGGTAGTGG \\
& qLAC-F & GGACTAATCTTGGGAATG \\
\hline
\end{tabular}



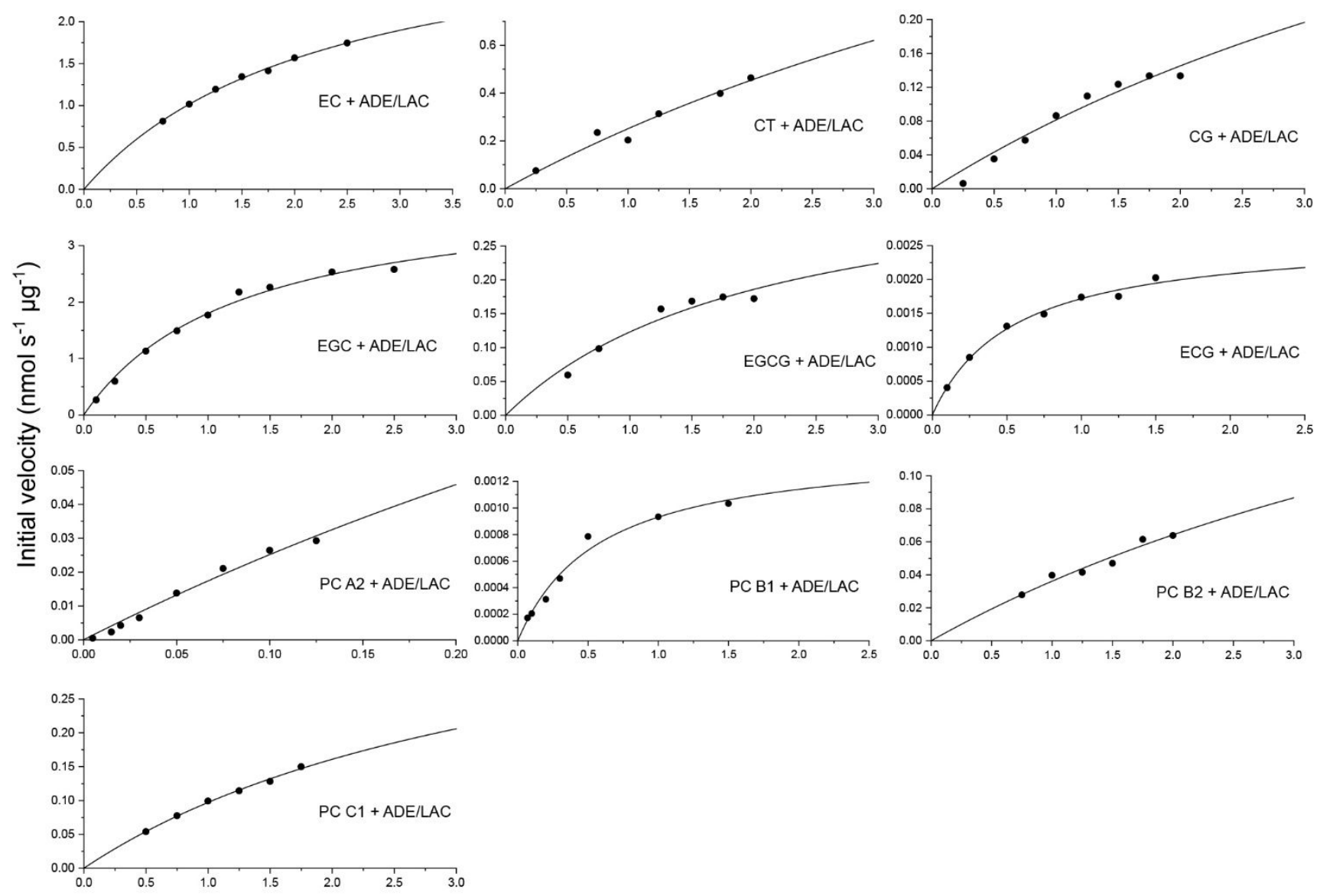

Substrate concentration (mM)

Figure S1. Non-linear regression plots for kinetic parameter assays of ADE/LAC on different flavonoid substrates. 

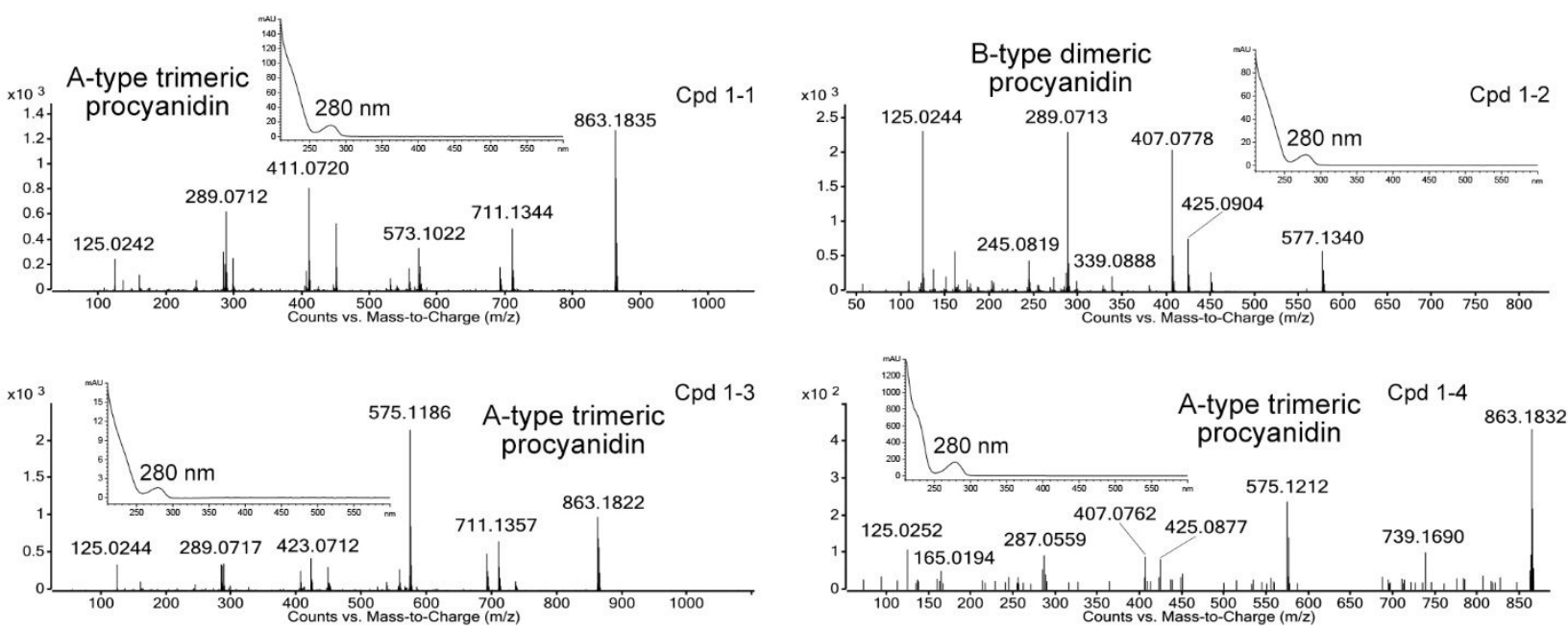

Figure S2. MS/MS spectra and UV-vis spectra of the 4 unknown PC oligomers in litchi pericarp. The 4 compounds were detected in the flavonoid extract from litchi pericarp at $0 \mathrm{~d}$ of the storage by HPLC and UPLC-DAD-QTOF-MS/MS. The HPLC profiles and the identification of the compounds based on MS/MS fragments were presented in Figure 2B and E. The maximum wavelength of the UV-Vis spectra for each detected product is indicated. 

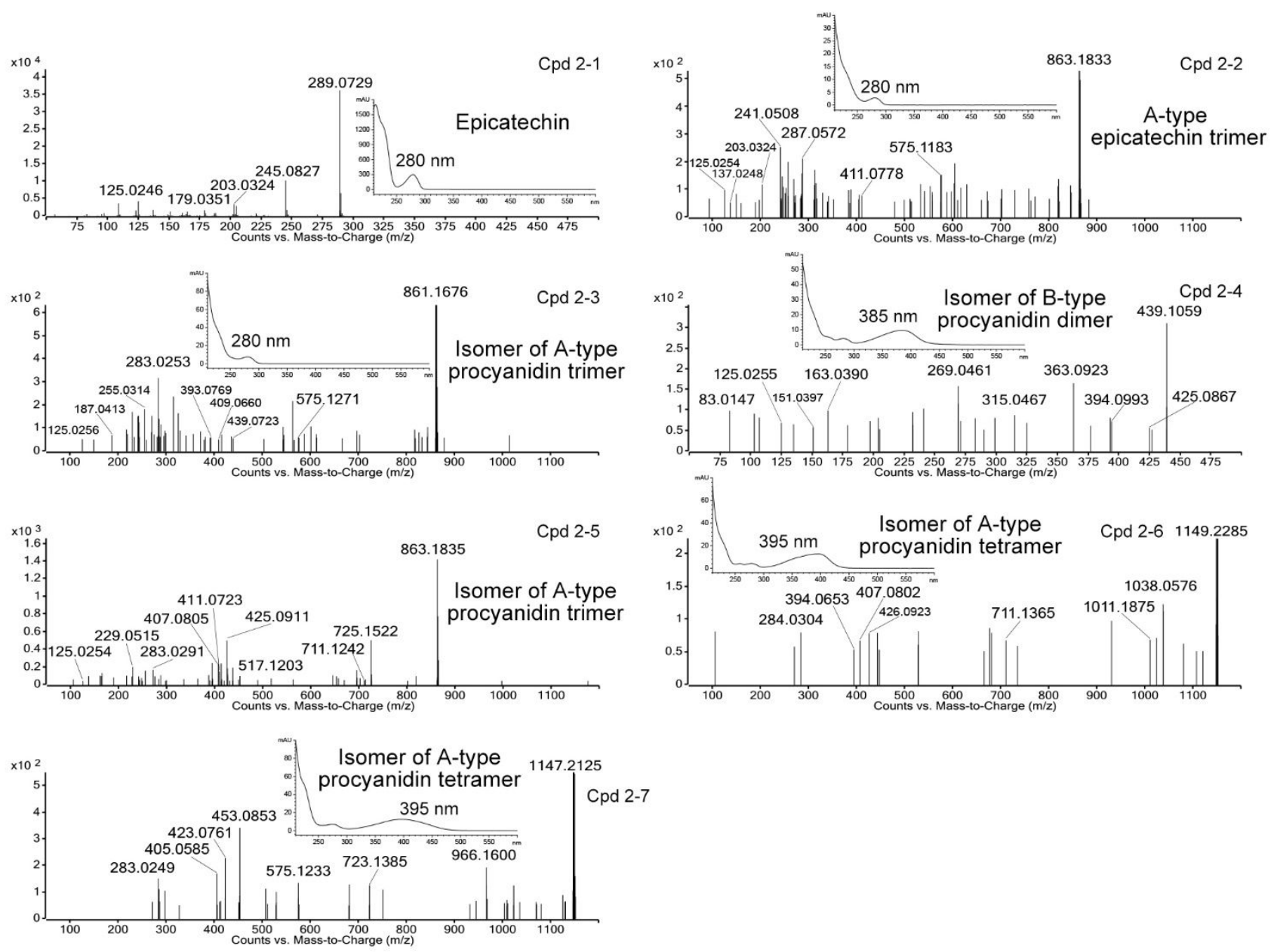

Figure S3. MS/MS spectra and UV-vis spectra of the main products detected in the in vitro EC reaction catalyzed by ADE/LAC. The chromatography profiles and identification of the products are presented in Figure 3C and E. The maximum wavelength of the UV-vis spectra for each detected product is indicated. 

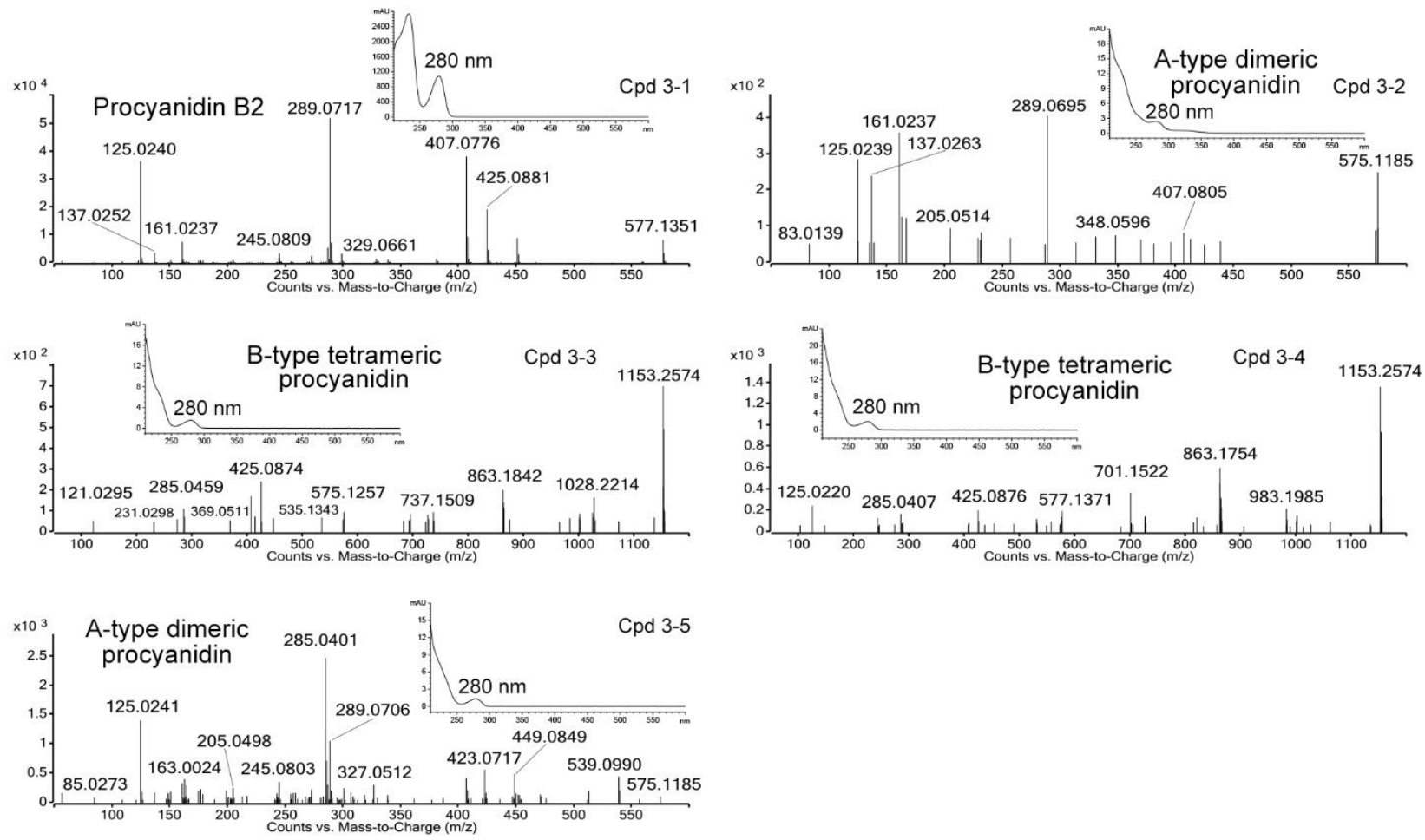

Figure S4. MS/MS spectra and UV-vis spectra of the main products detected in the in vitro PC B2 reaction of catalyzed by ADE/LAC. The chromatography profiles and identification of the products are presented in Figure 4B and C. The maximum wavelength of the UV-vis spectra for each detected product is indicated. 

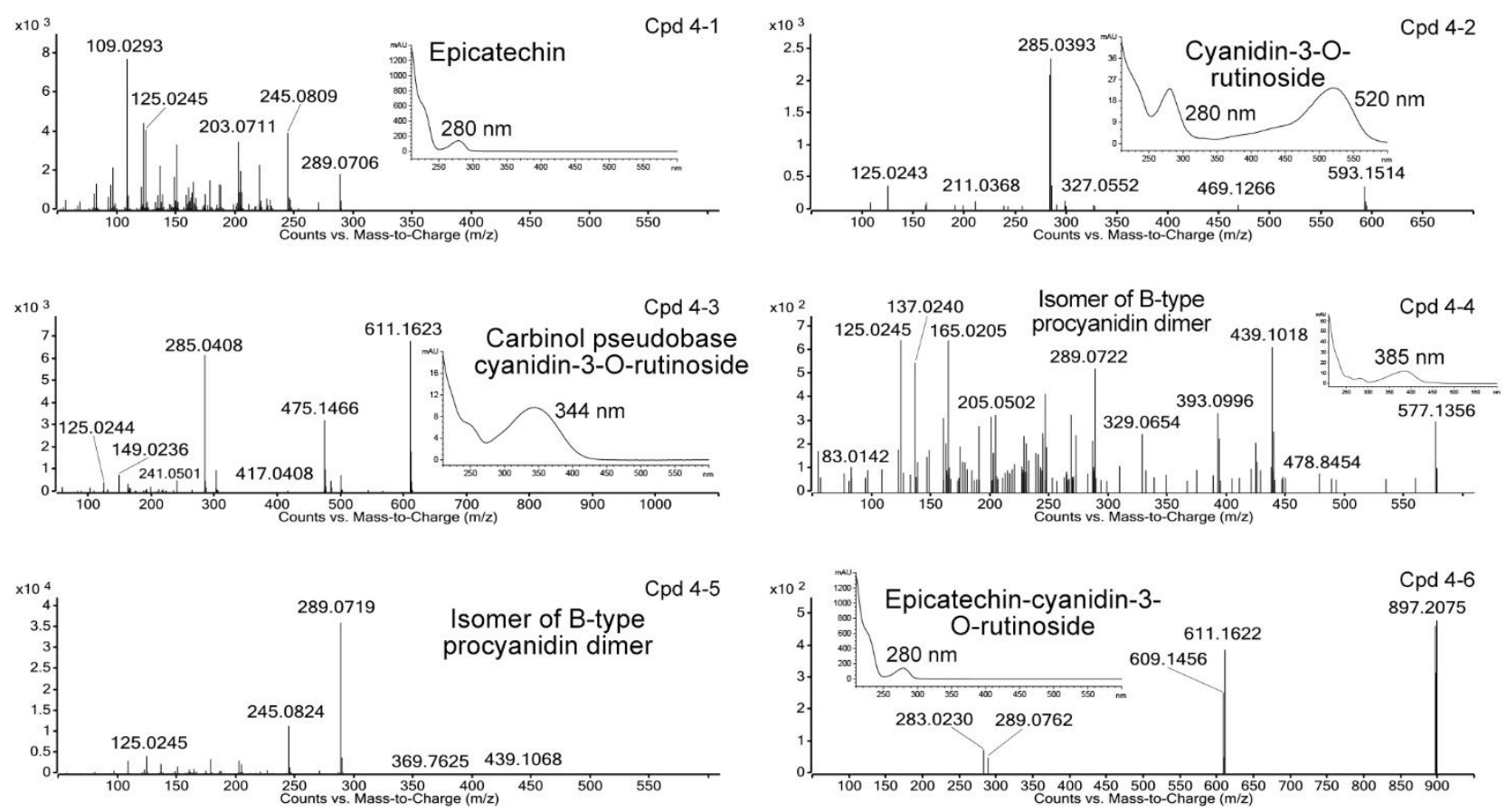

Figure S5. MS/MS spectra and UV-vis spectra of the main compounds detected in the reaction of EC and Cy3R catalyzed by ADE/LAC. The chromatography profiles and identification of the products are presented in Figure 6A and C. The maximum wavelength of the UV-vis spectra for each detected product is indicated, except for Cpd4-5 due to the failure to acquire the spectrum by UPLC-DAD-QTOF-MS/MS. 

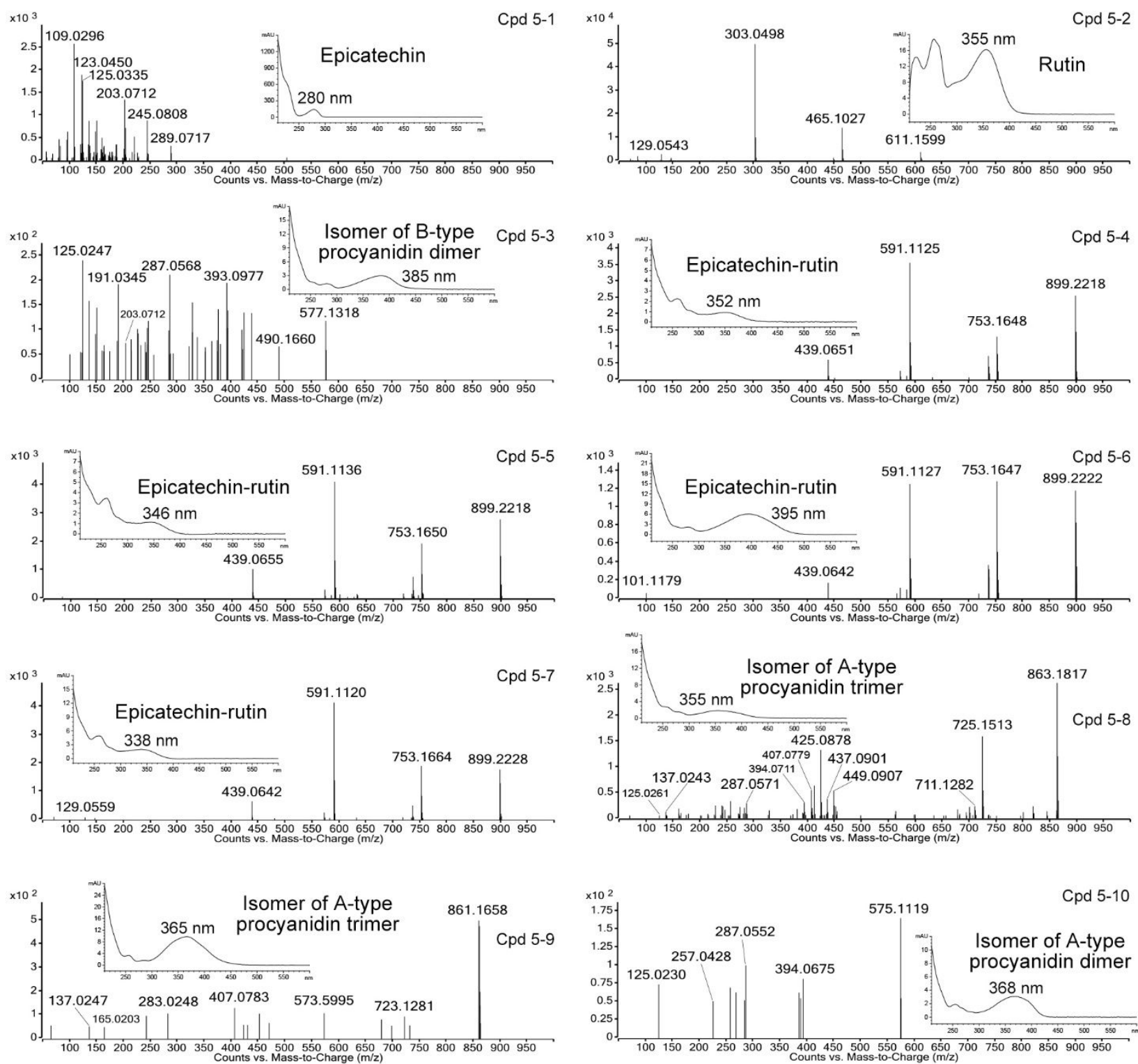

Figure S6. MS/MS spectra and UV-vis spectra of the main compounds detected in the reaction of EC and rutin catalyzed by $\mathrm{ADE} / \mathrm{LAC}$. The chromatography profiles and identification of the products are presented in Figure 6B and D. The maximum wavelength of the UV-vis spectra for each detected product is indicated. 